\title{
MULTIPLIERS OF IMPRIMITIVITY BIMODULES AND MORITA EQUIVALENCE OF CROSSED PRODUCTS
}

\author{
SIEGFRIED ECHTERHOFF and IAIN RAEBURN
}

The multiplier algebra $M(A)$ of a $C^{*}$-algebra $A$ is now recognised as an object of fundamental importance in operator algebras (see, e.g., [4], [9, §3.12]). Here we shall show that every $A-B$ imprimitivity bimodule $X$ has a multiplier bimodule $M(X)$ with analogous properties. Thus, by definition, $M(X)$ will consist of compatible pairs of maps $m_{A}: A \rightarrow X, m_{B}: B \rightarrow X$, representing left and right multiplication by the multiplier $m=\left(m_{A}, m_{B}\right)$; we shall characterise $M(X)$ as the universal $A-B$ bimodule $M$ containing $X$ as a submodule satisfying $A \cdot M \subset X$ and $M \cdot B \subset X$, just as $M(A)$ is the universal $C^{*}$-algebra containing $A$ as an essential ideal; and we shall show that appropriately nondegenerate homomorphisms of $X$ into another multiplier bimodule $M(Y)$ extend to strictly continuous homomorphisms of $M(X)$ into $M(Y)$. We shall also prove that we can simultaneously represent $A$ on a Hilbert space $\mathscr{H}, B$ on a Hilbert space $\mathscr{K}$, and $X$ as bounded operators from $\mathscr{K}$ to $\mathscr{H}$; if these representations are faithful, then $M(X)$ is faithfully represented as

$$
\{T \in B(\mathscr{K}, \mathscr{H}): a T \in X, T b \in X \text { for all } a \in A, b \in B\}
$$

(cf, for example, $[9,3.12 .3]$ ).

We were led to these ideas by our interest in recent work of Baaj-Skandalis [1] and Bui [3] on Morita equivalence of crossed products by coactions. A coaction of a locally compact group $G$ on a $C^{*}$-algebra $A$ is a nondegenerate homomorphism $\delta_{A}$ of $A$ into the multiplier algebra $M\left(A \otimes C_{r}^{*}(G)\right)$, satisfying several axioms (e.g. $[7, \S 2.1]$ ); a coaction of $G$ on an imprimitivity bimodule should therefore be, inter alia, a map $\delta_{X}$ of $X$ into $M\left(X \otimes C_{r}^{*}(G)\right)$. Baaj and Skandalis worked in the context of (one-sided) Hilbert modules, and used the space $\mathscr{L}_{B \otimes C_{r}^{*}(G)}\left(B \otimes C_{r}^{*}(G), X \otimes C_{r}^{*}(G)\right)$ of adjointable $B \otimes C_{r}^{*}(G)$-linear operators rather than $M\left(X \otimes C_{r}^{*}(G)\right)$. When Bui defined Morita equivalence of systems $\left(A, \delta_{A}\right)$ and $\left(B, \delta_{B}\right)$, he also used $\mathscr{L}_{B \otimes C_{r}^{*}(G)}\left(B \otimes C_{r}^{*}(G), X \otimes C_{r}^{*}(G)\right)$. However, in this situation, one might equally well view $X$ as a left Hilbert $A$-module and use $\mathscr{L}_{A \otimes C_{r}^{*}(G)}\left(A \otimes C_{r}^{*}(G), X \otimes C_{r}^{*}(G)\right)$, and it is not immediately clear that this gives

Received February 21, 1994. 
the same theory. Our Proposition 1.3 resolves this problem: for any $A-B$ imprimitivity bimodule $X, \mathscr{L}_{B}(B, X) \cong M(X) \cong \mathscr{L}_{A}(A, X)$.

Both Baaj-Skandalis and Bui proved that Morita equivalent systems $\left(A, \delta_{A}\right)$, $\left(B, \delta_{B}\right)$ have Morita equivalent crossed products, by showing that, if $X$ is an $A-B$ imprimitivity bimodule with a compatible coaction $\delta_{X}$ of $G$, then $X \otimes_{B}\left(B \times_{\delta_{B}} G\right)$ is an $A \times{ }_{\delta_{A}} G-B \times{ }_{\delta_{B}} G$ imprimitivity bimodule. This formulation has several disadvantages. First, the symmetry of the situation implies that $\left(A \times \delta_{\delta_{A}} G\right) \otimes_{A} X$ is also an $A \times \times_{\delta_{B}} G-B \times{ }_{\delta_{B}} G$ imprimitivity bimodule, but it is not obviously isomorphic to $X \otimes_{B}\left(B \times_{\delta_{B}} G\right)$, and, second, it is not clear how to describe the action of $A \times_{\delta_{A}} G$ on $X \otimes_{B}\left(B \times_{\delta_{B}} G\right)$. We shall obtain a symmetric version of this theorem, by representing $A$ on $\mathscr{H}, B$ on $\mathscr{K}$, and $X \otimes C_{r}^{*}(G)$ and its multiplier bimodule as operators in $B\left(\mathscr{K} \otimes L^{2}(G), \mathscr{H} \otimes L^{2}(G)\right)$; our $A \times_{\delta_{A}} G-B \times{\delta_{B}}_{B} G$ imprimitivity bimodule is then given by the space

$$
\overline{\mathrm{sp}}\left\{\delta_{X}(x)\left(1 \otimes M_{f}\right): x \in X, f \in C_{0}(G)\right\} \subset B\left(\mathscr{K} \otimes L^{2}(G), \mathscr{H} \otimes L^{2}(G)\right),
$$

with module actions and inner products defined using the corresponding actions of $A \times_{\delta_{A}} G$ and $B \times_{\delta_{B}} G$ on $\mathscr{H} \otimes L^{2}(G)$ and $\mathscr{K} \otimes L^{2}(G)$. (Of course, this turns out to be isomorphic to both $X \otimes_{B}\left(B \times_{\delta_{B}} G\right)$ and $\left(A \times_{\delta_{A}} G\right) \otimes_{A} X$.) We feel that our proof is more transparent because we have available the basic properties of multiplier bimodules, which help justify the complicated manipulations which are always necessary when dealing with coactions. As evidence that our formulation is also convenient, we use it to give a relatively short proof of Bui's main theorem, which is the corresponding Morita equivalence for the twisted systems of [10].

When dealing with Morita equivalence, one always has the choice of using the imprimitivity bimodule ${ }_{A} X_{B}$ or the linking algebra $L=\left(\begin{array}{ll}A & X \\ \tilde{X} & B\end{array}\right)$, and in an appendix we give a brief review of our theory in the context of linking algebras. We show that the multiplier bimodule $M(X)$ can be naturally identified with the top righthand corner in $M(L)$, and discuss how some of our results might be obtained from the analogous properties of $M(L)$. We have preferred to stick with bimodules in the body of the paper, largely because it is bimodules rather than linking algebras which arise in applications (see, for example, $[5,8,14])$, but also because we feel it gives a clearer picture of what is going on.

ACKNOWLEDGEMENT. This research was supported by grants from the University of Paderborn and the Australian Research Council.

\section{§1. The multiplier bimodule of an imprimitivity bimodule.}

In the following, let $A$ and $B$ be $C^{*}$-algebras, and suppose that $X$ is a complete $A-B$ imprimitivity bimodule. In other words, $X$ is an $A-B$ bimodule, a full left 
Hilbert $A$-module, a full right Hilbert $B$-module, such that $A$ and $B$ act as bounded operators on $X$, and the $A$ - and $B$-valued inner products ${ }_{A}\langle\cdot, \cdot\rangle$ and $\langle\cdot,\rangle_{B}$ satisfy the compatibility condition

$$
{ }_{A}\langle x, y\rangle \cdot z=x \cdot\langle y, z\rangle_{B} \text { for all } x, y, z \in X .
$$

We shall sometimes just say that ${ }_{A} X_{B}$ is an imprimitivity bimodule. We shall frequently use the canonical identifications of $A$ with the algebra $\mathscr{K}_{B}(X)$ of compact operators on the Hilbert $B$-module $X$, and that of $M(A)$ with $\mathscr{L}_{B}(X)$.

Definition 1.1. Suppose that $X$ is an $A-B$ imprimitivity bimodule. A multiplier of $X$ is a pair $m=\left(m_{A}, m_{B}\right)$, where $m_{A}: A \rightarrow X$ is $A$-linear, $m_{B}: B \rightarrow X$ is $B$-linear, and

$$
m_{A}(a) \cdot b=a \cdot m_{B}(b) \text { for all } a \in A, b \in B .
$$

We write $M(X)$ for the set of all multipliers of $X$.

As with double centralizers of a $C^{*}$-algebra, we should think of $m_{A}$ as the map $a \mapsto a \cdot m, m_{B}$ as the map $b \mapsto m \cdot b$, and (1.1) as the commuting of the module actions. To make this precise, note that every $x \in X$ can be viewed as a multiplier of $X$ via the actions of $A$ and $B$ on $X$; in other words, $x \mapsto\left(x_{A}, x_{B}\right)$, where $x_{A}(a)=$ $a \cdot x, x_{B}(b)=x \cdot b$, is a map of $X$ into $M(X)$, and this is an injection because $a \cdot x=0$ for all $a$ implies

$$
0=\|_{A}\left\langle\left\langle_{A}\langle x, x\rangle \cdot x, x\right\rangle\|=\|_{A}\langle x, x\rangle^{*}\langle x, x\rangle\|=\| x \|^{4} .\right.
$$

We use this injection to identify $X$ with a subspace of $M(X)$. In particular, this allows us to define actions of $A$ and $B$ on $M(X)$ by $a \cdot m=m_{A}(a), m \cdot b=m_{B}(b)$, and then $M(X)$ is an $A-B$ bimodule. We therefore refer to $M(X)$ as the multiplier bimodule of $X$. It can be characterised alternatively as follows:

Proposition 1.2. Let $X$ be an $A-B$ imprimitivity bimodule. Then $M(X)$ is an $A-B$ bimodule which satisfies the following two conditions:

(1) $A \cdot M(X) \subseteq X$ and $M(X) \cdot B \subseteq X$.

(2) If $M$ is any other $A-B$ bimodule which contains $X$ and satisfies (l), then there exists a unique bimodule homomorphism $M \rightarrow M(X)$ which is the identity on $X$. Moreover, any $A-B$ bimodule which contains $X$ and which satisfies Conditions (1) and (2) is isomorphic (as an $A-B$ bimodule) to $M(X)$.

Proof. Property (1) follows immediately from the definition of the module actions on $M(X)$. If $M$ is any other $A-B$ bimodule which contains $X$ and satisfies (1), then we define $\Phi: M \rightarrow M(X)$ by $\Phi(m)=\left(\Phi(m)_{A}, \Phi(m)_{B}\right)$ with $\Phi(m)_{A}(a)=a \cdot m$ and $\Phi(m)_{B}(b)=m \cdot b$, and $\Phi$ is a bimodule homomorphism which is the identity on $X$. Suppose now that $\Psi: M \rightarrow M(X)$ is another bimodule homomorphism 
which fixes $X$. Then $\left(\Phi(m)_{A}-\Psi(m)_{A}\right)(a)=a \cdot m-\Psi(a \cdot m)_{A}=0$, and similarly $\Psi(m)_{B}=\Phi(m)_{B}$. Thus $\Psi=\Phi$.

Suppose now that $M$ is an $A-B$ bimodule which contains $X$ and which satisfies Conditions (1) and (2). Then there exist unique $X$-fixing bimodule homomorphisms $\Phi: M \rightarrow M(X)$ and $\Psi: M(X) \rightarrow M$. The uniqueness condition implies that $\Psi \circ \Phi$ is the identity on $M$ and $\Phi \circ \Psi$ is the identity on $M(X)$.

We denote by $\mathscr{L}_{B}(B, X)$ the set of all $B$-linear operators $T: B \rightarrow X$ which are adjointable, i.e., for which there is a $B$-linear map $T^{*}: X \rightarrow B$ such that

$$
\langle T(b), x\rangle_{B}=b^{*} T^{*}(x) \text { for all } b \in B, x \in X .
$$

It is shown in [6, p. 4] that every $T \in \mathscr{L}_{B}(B, X)$ is automatically bounded. There are canonical left and right actions of $A$ and $B$ on $\mathscr{L}_{B}(B, X)$ given by

$$
(a \cdot T)(c)=a \cdot(T(c)) \text { and }(T \cdot b)(c)=T(b c) .
$$

Moreover, the left action of $B$ gives a canonical embedding $x \mapsto T_{x}$ of $X$ into $\mathscr{L}_{B}(B, X)$, i.e., $T_{x}(b)=x \cdot b$ for $b \in B$; the adjoint is given by $T_{x}^{*}(y)=\langle x, y\rangle_{B}$. Of course, the actions of $A$ and $B$ on the subspace $X$ of $\mathscr{L}_{B}(B, X)$ agree with the usual ones, so we can view $X$ as an $A-B$ submodule of $\mathscr{L}_{B}(B, X)$.

Similarly, if $\mathscr{L}_{A}(A, X)$ denotes the set of all $A$-linear adjointable maps from $A$ to $X$, then we have actions of $A$ and $B$ on $\mathscr{L}_{A}(A, X)$ and $X$ embeds as a submodule of $\mathscr{L}_{A}(A, X)$.

Proposition 1.3. Let $X$ be an $A-B$ imprimitivity bimodule. Then $\mathscr{L}_{B}(B, X)$ and $\mathscr{L}_{A}(A, X)$ satisfy the conditions of Proposition 1.2. Indeed, the maps $m \mapsto m_{B}$ : $M(X) \rightarrow \mathscr{L}_{B}(B, X)$ and $m \mapsto m_{A}: M(X) \rightarrow \mathscr{L}_{A}(A, X)$, are bimodule isomorphisms which are the identities on the embedded copies of $X$.

For the proof of Proposition 1.3 we need:

Lemma 1.4. Let $m \in M(X)$. Then $m_{B} \in \mathscr{L}_{B}(B, X)$, with $m_{B}^{*}(x)$ given by the unique element of $B$ satisfying

$$
m_{A}\left({ }_{A}\langle z, x\rangle\right)=z \cdot\left(m_{B}^{*}(x)\right)^{*} \text { for all } z \in X .
$$

Similarly, $m_{A} \in \mathscr{L}_{A}(A, X)$, with $m_{A}^{*}(x)$ characterized by

$$
\left(m_{A}^{*}(x)\right)^{*} \cdot z=m_{B}\left(\langle x, z\rangle_{B}\right) \text { for all } z \in X .
$$

Moreover, $m_{B}^{*}(a \cdot x)=\left\langle m_{A}\left(a^{*}\right), x\right\rangle_{B}$ and $m_{A}^{*}(x \cdot b)={ }_{A}\left\langle x, m_{B}\left(b^{*}\right)\right\rangle$.

Proof. By symmetry, it is enough to prove the statements for $m_{B}^{*}$. We first observe that for each $y \in X$ the map $z \rightarrow m_{A}\left({ }_{A}\langle z, y\rangle\right)$ is a compact $A$-linear operator on $X$; to see this, factor $y=a \cdot x$ for $a \in A$ and $x \in X$, and observe that $m_{A}\left({ }_{A}\langle z, a \cdot x\rangle\right)=m_{A}\left({ }_{A}\langle z, x\rangle a^{*}\right)={ }_{A}\langle z, x\rangle \cdot m_{A}\left(a^{*}\right)$. Since $B=\mathscr{K}_{A}(X)$, it follows 
that there exists a unique element $c \in B$ such that $z \cdot c=m_{A}\left({ }_{A}\langle z, y\rangle\right)$ for all $z \in X$, and we can indeed define a map $m_{B}^{*}: X \rightarrow B$ by (1.2). For $b \in B$ and $z \in X$, we have

$$
z \cdot\left(m_{B}^{*}(y)^{*} b\right)=m_{A}\left({ }_{A}\langle z, y\rangle\right) \cdot b={ }_{A}\langle z, y\rangle \cdot m_{B}(b)=z \cdot\left\langle y, m_{B}(b)\right\rangle_{B},
$$

which implies that $m_{B}^{*}$ is an adjoint of $m_{B}$. Finally,

$$
z \cdot\left(m_{B}^{*}(a \cdot x)\right)^{*}=m_{A}\left({ }_{A}\langle z, a \cdot x\rangle\right)={ }_{A}\langle z, x\rangle \cdot m_{A}\left(a^{*}\right)=z \cdot\left\langle x, m_{A}\left(a^{*}\right)\right\rangle_{B},
$$

and hence $m_{B}^{*}(a \cdot x)=\left\langle m_{A}\left(a^{*}\right), x\right\rangle_{B}$.

Proof of Proposition 1.3. We show that $\mathscr{L}_{B}(B, X)$ satisfies Conditions (1) and (2) of Proposition 1.2. For the first, suppose that $T \in \mathscr{L}_{B}(B, X)$. Then we immediately have $T \cdot b=T(b) \in X$. To prove that $a \cdot T \in X$ for $a \in A$, note that because the module action of $A$ on $\mathscr{L}_{B}(B, X)$ is continuous, it is enough to consider $a$ of the form ${ }_{A}\langle x, y\rangle$. But then the equation

$$
\left({ }_{A}\langle x, y\rangle \cdot T\right)(b)={ }_{A}\langle x, y\rangle \cdot T(b)=x \cdot\langle y, T(b)\rangle_{B}=x \cdot\left(T^{*}(y)\right)^{*} b
$$

implies that $a \cdot T=x \cdot\left(T^{*}(y)\right)^{*}$ is in $X$.

To verify (2), suppose that $M$ is an $A-B$ bimodule containing $X$ such that $A \cdot M \subseteq X$ and $M \cdot B \subseteq X$. We have to show that there is a unique bimodule homomorphism $\Phi: M \rightarrow \mathscr{L}_{B}(B, X)$ such that $\Phi(x)=T_{x}$ for $x \in X$. The uniqueness is easy: if $\Phi$ and $\Psi$ agree on $X$, then for $m \in M$ we have $\Phi(m)(b)=$ $\Phi(m) \cdot b=\Phi(m \cdot b)=\Psi(m \cdot b)=\Psi(m)(b)$ for all $b$, which implies $\Phi(m)=\Psi(m)$. To construct $\Phi$, note that left and right multiplication by $m \in M$ define a multiplier $\left(m_{A}, m_{B}\right)$ of $X$. Then Lemma 1.4 shows that $m_{B} \in \mathscr{L}_{B}(B, X)$, and we can define $\Phi(m)=m_{B}$, i.e. $\Phi(m)(b)=m \cdot b$.

Notice that if we started with $M=M(X), \Phi(m)$ is by definition just $m_{B}$, and since $M(X)$ has the same universal properties, $\Phi$ is an isomorphism. (See the last paragraph in the proof of Proposition 1.2.) Similarly, $m_{A}$ is an isomorphism of $M(X)$ onto $\mathscr{L}_{A}(A, X)$.

DEFINITION 1.5. Let ${ }_{A} X_{B}$ be an imprimitivity bimodule. The strict topology on the multiplier bimodule $M(X)$ is the topology generated by the seminorms $m \mapsto\|m \cdot b\|=\left\|m_{B}(b)\right\|$ and $m \mapsto\|a \cdot m\|=\left\|m_{A}(a)\right\|$.

REMARK. Let $\left(u_{i}\right)_{i \in I}$ be an approximate identity of $B$ satisfying $u_{i}=u_{i}^{*}$ and $\left\|u_{i}\right\|=1$. Because $m_{A}$ and $m_{B}$ are bounded linear maps, [6, Lemma 1.1.4] implies that $m \cdot u_{i}$ converges strictly to $m \in M(X)$. Thus $X$ is strictly dense in $M(X)$.

Furthermore, $M(X)$ is complete with respect to the strict topology. To see this, assume that $\left(m_{i}\right)_{i \in I}$ is a strictly Cauchy net in $M(X)$, so that for all $a \in A$ and $b \in B$, $\left(a \cdot m_{i}\right)_{i \in I}$ and $\left(m_{i} \cdot b\right)_{i \in I}$ are Cauchy nets in $X$ with respect to the norm topology. If we define $a \cdot m=\lim _{i \in I} a \cdot m_{i}$ and $m \cdot b=\lim _{i \in I} m_{i} \cdot b$, then $m \in M(X)$ and $\left(m_{i}\right)_{i \in I}$ converges strictly to $m$. 
Proposition 1.6. Let ${ }_{A} X_{B}$ be an imprimitivity bimodule. The module actions and inner products on $X$ extend to separately strictly continuous pairings

$$
\begin{gathered}
M(A) \times M(X) \rightarrow M(X), \quad M(X) \times M(B) \rightarrow M(X) \text { and } \\
M(A)\langle\cdot,\rangle: M(X) \times M(X) \rightarrow M(A),\langle\cdot,\rangle_{M(B)}: M(X) \times M(X) \rightarrow M(B) .
\end{gathered}
$$

Then $M(X)$ is a left $M(A)$-Hilbert module, right $M(B)$-Hilbert module satisfying $l \cdot\langle m, n\rangle_{M(B)}={ }_{M(A)}\langle l, m\rangle \cdot n$. However, $M(X)$ is not always an $M(A)-M(B)$ imprimitivity bimodule.

For the proof we need two lemmas.

LEMMA 1.7. The isomorphisms $m \mapsto m_{A}$ and $m \mapsto m_{B}$ of $M(X)$ onto $\mathscr{L}_{A}(A, X)$ and $\mathscr{L}_{B}(B, X)$, respectively, are continuous with respect to the strict topology on $M(X)$ and the *-strong topologies on $\mathscr{L}_{A}(A, X)$ and $\mathscr{L}_{B}(B, X)$, respectively.

ProOF. Suppose that $m^{i}$ converges strictly to $m$ in $M(X)$. Then it follows from the definition of the strict topology that $m_{B}^{i}(b)$ converges to $m_{B}(b)$ in norm for all $b \in B$. To see that $\left(m_{B}^{i}\right)^{*}(y)$ converges to $m_{B}^{*}(y)$ for all $y$, write $y=a \cdot x$. Then using Lemma 1.4 we have $\left(m_{B}^{i}\right)^{*}(a \cdot x)=\left\langle m_{A}^{i}\left(a^{*}\right), x\right\rangle_{B}$, and this converges to $\left\langle m_{A}\left(a^{*}\right), x\right\rangle_{B}=m_{B}^{*}(a \cdot x)$ by the continuity of $\langle\cdot, \cdot\rangle_{B}$.

LEMMA 1.8. Let ${ }_{A} X_{B}$ be an imprimitivity bimodule and let $m, n \in M(X)$. Using the canonical identifications of $\mathscr{L}_{B}(B), \mathscr{L}_{A}(X)$ with $M(B)$ and $\mathscr{L}_{A}(A), \mathscr{L}_{B}(X)$ with $M(A)$, we have

$$
m_{B}^{*} \circ n_{B}=n_{A} \circ m_{A}^{*} \text { in } M(B) \text { and } m_{B} \circ n_{B}^{*}=n_{A}^{*} \circ m_{A} \text { in } M(A) .
$$

Proof. By symmetry, it is enough to prove the identity $m_{B}^{*} \circ n_{B}=n_{A} \circ m_{A}^{*}$. Let $x \in X$ and $b \in B$. Then from several applications of Lemma 1.4 and the equation $\left(n_{A} \circ m_{A}^{*}\right)^{*}=m_{A} \circ n_{A}^{*}$ we obtain

$$
\begin{aligned}
x \cdot\left(m_{B}^{*} \circ n_{B}(b)\right)^{*} & =x \cdot\left(m_{B}^{*}\left(n_{B}(b)\right)\right)^{*}=m_{A}\left({ }_{A}\left\langle x, n_{B}(b)\right\rangle\right) \\
& =m_{A}\left(n_{A}^{*}\left(x \cdot b^{*}\right)\right)=x \cdot b^{*}\left(m_{A} \circ n_{A}^{*}\right) \\
& =x \cdot b^{*}\left(n_{A} \circ m_{A}^{*}\right)^{*}=x \cdot\left(\left(n_{A} \circ m_{A}^{*}\right) b\right)^{*},
\end{aligned}
$$

which implies $m_{B}^{*} \circ n_{B}=n_{A} \circ m_{A}^{*}$.

Proof of Proposition 1.6. Define the pairing $(k, m) \mapsto k \cdot m$ of $M(A) \times M(X)$ into $M(X)$ by

$$
(k \cdot m)_{A}(a)=m_{A}(a k) \text { and }(k \cdot m)_{B}(b)=k \cdot m_{B}(b) .
$$

Then $(k \cdot m)_{A}(a) \cdot b=m_{A}(a k) \cdot b=a k \cdot m_{B}(b)=a \cdot(k \cdot m)_{B}(b)$ for all $a$ and $b$, which implies $k \cdot m \in M(X)$. A quick calculation shows that this pairing extends the left action of $A$ on $X$ to a left action of $M(A)$ on $M(X)$. Assume that $k_{i}$ converges 
strictly to $k$ in $M(A)$. By factoring $x=a \cdot y$, we can see that $k_{i} \cdot x$ converges in norm to $k \cdot x$ for all $x \in X$, and this in turn implies that $k_{i} \cdot m$ converges strictly to $k \cdot m$ in $M(X)$. Similarly, if $m_{i}$ converges strictly to $m$, then $k \cdot m_{i}$ converges strictly to $k \cdot m$. The same arguments show that the pairing $M(X) \times M(B) \rightarrow M(X)$ defined by

$$
(m \cdot l)_{A}(a)=m_{A}(a) \cdot l \text { and }(m \cdot l)_{B}(b)=m_{B}(l b), \quad l \in M(B),
$$

is separately strictly continuous and extends the action of $B$ on $X$.

We define the inner products ${ }_{M(A)}\langle\cdot, \cdot\rangle$ and $\langle\cdot, \cdot\rangle_{M(B)}$ on $M(X)$ by

$$
\begin{aligned}
& { }_{M(A)}\langle m, n\rangle=m_{B}{ }^{\circ} n_{B}^{*} \in \mathscr{L}_{B}(X)=M(A) \text {, and } \\
& \langle m, n\rangle_{M(B)}=m_{B}^{*} \circ n_{B} \in \mathscr{L}_{B}(B)=M(B) .
\end{aligned}
$$

If $x, y \in X \subseteq M(X)$, then $x_{B} \circ y_{B}^{*}(z)=x_{B}\left(\langle y, z\rangle_{B}\right)=x \cdot\langle y, z\rangle_{B}={ }_{A}\langle x, y\rangle \cdot z$ for all $z \in X$, which implies ${ }_{M(A)}\langle x, y\rangle={ }_{A}\langle x, y\rangle$. Similarly, $x_{B}^{*} \circ y_{B}(b)=\langle x, y \cdot b\rangle_{B}=$ $\langle x, y\rangle_{B} b$ implies $\langle x, y\rangle_{M(B)}=\langle x, y\rangle_{B}$. Thus ${ }_{M(A)}\langle\cdot, \cdot\rangle$ and $\langle\cdot, \cdot\rangle_{M(B)}$ do extend the $A$ - and $B$-valued inner products on $X$. If $m^{i}$ converges strictly to $m$, then $\left\langle m^{i}, n\right\rangle_{M(B)} b=\left(m_{B}^{i}\right)^{*}\left(n_{B}(b)\right)$ converges in norm to $m_{B}^{*}\left(n_{B}(b)\right)=\langle m, n\rangle_{M(B)} b$ by Lemma 1.7. On the other hand,

$$
\left(b\left\langle m^{i}, n\right\rangle_{M(B)}\right)^{*}=\left(\left(m_{B}^{i}\right)^{*} \circ n_{B}\right)^{*} b^{*}=\left(n_{B}^{*} \circ m_{B}^{i}\right) b^{*}=n_{B}^{*}\left(m_{B}^{i}(b)\right),
$$

which converges to $n_{B}^{*}\left(m_{B}(b)\right)=\left(b\langle m, n\rangle_{M(B)}\right)^{*}$ by the norm continuity of $n_{B}^{*}$. Thus $\left\langle m^{i}, n\right\rangle_{M(B)} \rightarrow\langle m, n\rangle_{M(B)}$ strictly, and since

$$
\langle m, n\rangle_{M(B)}^{*}=\left(m_{B}^{*} \circ n_{B}\right)^{*}=n_{B}^{*} \circ m_{B}=\langle n, m\rangle_{M(B)},
$$

this implies that $\langle\cdot,\rangle_{M(B)}$ is separately strictly continuous. The separate strict continuity of ${ }_{M(A)}\langle\cdot, \cdot\rangle$ now follows from Lemma 1.8 by applying similar arguments to the expressions $n_{A} \circ m_{A}^{*}$ and $n_{A}^{*} \circ m_{A}$.

Since $m_{B}^{*} \circ m_{B}$ and $m_{B} \circ m_{B}^{*}$ are zero if and only if $m_{B}=0$, the inner products are positive definite. By writing the positive element $m_{B}^{*} \circ m_{B}$ as $d^{2}$ for some $d \in M(B)^{+}$, and computing the norm of $\left\|d^{2}\right\|$ as $\sup _{\|b\| \leqq 1}\left\|(d b)^{*} d b\right\|$, it is easily checked that $\left\|m_{B}^{*} \circ m_{B}\right\|=\left\|m_{B}\right\|_{\mathscr{L}_{B}(B, X)}^{2}$. Thus $\|m\|_{M(B)}^{2}=\left\|m_{B}^{*} \circ m_{B}\right\|=$ $\left\|m_{B}\right\|_{\mathscr{L}_{B}(B, X)}^{2}=\left\|m_{B} \circ \mathrm{m}_{B}^{*}\right\|={ }_{M(A)}\|m\|^{2}$. Because $\mathscr{L}_{B}(B, X)$ is complete in the operator norm, the first two equations imply that $M(X)$ is complete in the norm given by the inner products. That $M(X)$ is a left $M(A)$-and a right $M(B)$-Hilbert module now follows from the separate strict continuity of the operations and the strict density of $X, A$ and $B$ in $M(X), M(A)$ and $M(B)$. Similarly, we have $l \cdot\langle m, n\rangle_{M(B)}=$ ${ }_{M(A)}\langle l, m\rangle \cdot n$ for $l, m, n \in M(X)$. For the last assertion, see the remark below.

ReMARK. For any $A-B$ imprimitivity bimodule $X$, denote by $R(A)$ and $R(B)$ the closed linear spans of $\left\{{ }_{M(A)}\langle m, n\rangle: m, n \in M(X)\right\}$ and $\left\{\langle m, n\rangle_{M(B)}: m, n \in M(X)\right\}$ in $M(A)$ and $M(B)$, respectively. Proposition 1.6 says that $M(X)$ is an $R(A)-R(B)$ 
imprimitivity bimodule having $X$ as an $A-B$ imprimitivity submodule. If $A$ or $B$ is unital, then Proposition 1.3 implies that $M(X)=X, R(A)=A$ and $R(B)=B$. For example, if we regard a Hilbert space $\mathscr{H}$ as a $K(\mathscr{H})$-C imprimitivity bimodule, then $M(\mathscr{H})=\mathscr{H}$. Thus $M(X)$ is in general not an $M(A)-M(B)$ imprimitivity bimodule. On the other hand, it can be: if we view a $C^{*}$-algebra $A$ as an $A$ - $A$ imprimitivity bimodule, then the multiplier bimodule of $A$ coincides with the multiplier algebra $M(A)$ and we have $R(A)=M(A)$ on either side. In Example 2.5 below we describe an $A-B$ imprimitivity bimodule $X$ such that $A \neq$ $R(A) \neq M(A)$.

Recall that for any pair of $C^{*}$-algebras $B$ and $D$, a homomorphism $\Phi: B \rightarrow$ $M(D)$ is called nondegenerate if $\Phi(B) D$ is dense in $D$. If $\Phi$ is nondegenerate, then $\Phi$ extends uniquely to a strictly continuous homomorphism $\bar{\Phi}: M(B) \rightarrow M(D)$ (e.g. [7, Lemma 1.1]). We now show that a similar result holds for multiplier bimodules.

DEFINITION 1.8. An imprimitivity-bimodule homomorphism $\Phi:{ }_{A} X_{B} \rightarrow M\left({ }_{C} Y_{D}\right)$ is a triple $\Phi=\left(\Phi_{A}, \Phi_{X}, \Phi_{B}\right)$, in which $\Phi_{A}: A \rightarrow M(C)$ and $\Phi_{B}: B \rightarrow M(D)$ are homomorphisms, and $\Phi_{X}: X \rightarrow M(Y)$ is a linear map satisfying the following compatibility conditions:

(1) $\Phi_{A}\left({ }_{A}\langle x, y\rangle\right)={ }_{M(C)}\left\langle\Phi_{X}(x), \Phi_{X}(y)\right\rangle, \Phi_{B}\left(\langle x, y\rangle_{B}\right)=\left\langle\Phi_{X}(x), \Phi_{X}(y)\right\rangle_{M(D)}$ for all $x, y \in X$, and

(2) $\Phi_{X}(a \cdot x \cdot b)=\Phi_{A}(a) \cdot \Phi_{X}(x) \cdot \Phi_{B}(b)$ for all $a \in A, x \in X$ and $b \in B$.

We say that $\Phi$ is nondegenerate if $\Phi_{A}$ and $\Phi_{B}$ are nondegenerate.

Proposition 1.9. Let $\Phi=\left(\Phi_{A}, \Phi_{X}, \Phi_{B}\right): X \rightarrow M(Y)$ be a nondegenerate imprimitivity-bimodule homomorphism. Then there exists a unique strictly continuous extension $\bar{\Phi}=\left(\bar{\Phi}_{A}, \bar{\Phi}_{X}, \bar{\Phi}_{B}\right): M(X) \rightarrow M(Y)$, and the compatibility conditions of Definition 1.8 are still satisfied.

ProOF. Let $\bar{\Phi}_{A}$ and $\bar{\Phi}_{B}$ denote the unique strictly continuous extensions of $\Phi_{A}$ and $\Phi_{B}$ to $M(A)$ and $M(B)$, respectively. By Cohen's factorisation theorem we have $D=\Phi_{B}(B) D$ and $C=C \Phi_{A}(A)$. Let $m \in M(X), c \Phi_{A}(a) \in C$ and $\Phi_{B}(b) d \in D$. Then we aim to define

$$
\bar{\Phi}_{X}(m)_{C}\left(c \Phi_{A}(a)\right)=c \cdot \Phi_{X}(a \cdot m) \text { and } \bar{\Phi}_{X}(m)_{D}\left(\Phi_{B}(b) d\right)=\Phi_{X}(m \cdot b) \cdot d .
$$

To see, for example, that $\bar{\Phi}_{X}(m)_{D}$ is a well defined map of $D$ into $Y$, observe that the right-hand side is bilinear in $(b, d)$ and $B$-balanced, hence determines a linear map of $B \otimes_{B} D$ into $Y$. Since $b \otimes d \rightarrow \Phi_{B}(b) d$ is an isomorphism of $B \otimes_{B} D$ onto $D$, it follows that $\bar{\Phi}_{X}(m)_{D}$ is well defined.

Routine calculations show that $\bar{\Phi}_{X}$ is strictly continuous, which implies that $\bar{\Phi}_{X}$ satisfies the various compatibility conditions. The uniqueness of the strictly continuous extension follows from the strict density of $X$ in $M(X)$. 
EXAMPLE 1.10. Suppose that ${ }_{A} X_{B}$ is an imprimitivity bimodule, and that $\Phi_{B}$ : $B \rightarrow M(D)$ is a nondegenerate homomorphism for some $C^{*}$-algebra $D$. Let $C$ be the algebra $\mathscr{K}_{D}\left(X \otimes_{B} D\right)$ of compact operators on the Hilbert $D$-module $X \otimes_{B} D$. The left action of $A$ on $X$ defines a nondegenerate homomorphism $\Phi_{A}: A \rightarrow$ $M(C)=\mathscr{L}_{D}\left(X \otimes_{B} D\right)$. We define $\Phi_{X}: X \rightarrow M\left(X \otimes_{B} D\right) \cong \mathscr{L}_{D}\left(D, X \otimes_{B} D\right)$ by $\Phi_{X}(x)_{D}(d)=x \otimes d$. (This is adjointable because $\Phi_{X}(x)_{D}^{*}(y \otimes e)=\Phi_{B}\left(\langle x, y\rangle_{B}\right) e$ defines an adjoint.) Some quick calculations show that $\left(\Phi_{A}, \Phi_{X}, \Phi_{B}\right): X \rightarrow$ $M\left(X \otimes_{B} D\right)$ is an imprimitivity-bimodule homomorphism.

Assume now also that ${ }_{C} Y_{D}$ is an imprimitivity bimodule, and that there are a nondegenerate homomorphism $\Phi_{B}: B \rightarrow M(D)$ and a linear map $\Phi_{X}: X \rightarrow$ $M(Y)$ such that $\Phi_{B}\left(\langle x, y\rangle_{B}\right)=\left\langle\Phi_{X}(x), \Phi_{X}(y)\right\rangle_{M(D)}, \Phi_{X}(x \cdot b)=\Phi_{X}(x) \Phi_{B}(b)$, and $\Phi_{X}(X) D$ is dense in $Y$. Then the map $x \otimes d \mapsto \Phi_{X}(x) d$ extends to an isomorphism $X \otimes_{B} D \cong Y$ of Hilbert $D$-modules, and it follows that there is a nondegenerate homomorphism $\Phi_{A}: A \rightarrow M(C)$ such that $\left(\Phi_{A}, \Phi_{X}, \Phi_{B}\right): X \rightarrow M(Y)$ is an imprimitivity-bimodule homomorphism.

\section{§2. Representations of imprimitivity bimodules.}

The main purpose of this section is to show that every imprimitivity bimodule ${ }_{A} X_{B}$ may be represented faithfully as operators on Hilbert space.

Definition 2.1. A representation of an $A-B$ imprimitivity bimodule $X$ on the pair of Hilbert spaces $(\mathscr{H}, \mathscr{K})$ is a triple $\left(\pi_{A}, \pi_{X}, \pi_{B}\right)$ consisting of nondegenerate representations $\pi_{A}: A \rightarrow B(\mathscr{H}), \pi_{B}: B \rightarrow B(\mathscr{K})$, and a linear map $\pi_{X}: X \rightarrow$ $B(\mathscr{K}, \mathscr{H})$ satisfying

(1) $\pi_{X}(x)^{*} \pi_{X}(y)=\pi_{B}\left(\langle x, y\rangle_{B}\right)$ and $\pi_{X}(x) \pi_{X}(y)^{*}=\pi_{A}\left({ }_{A}\langle x, y\rangle\right)$ for $x, y \in X$.

(2) $\pi_{X}(a \cdot x \cdot b)=\pi_{A}(a) \pi_{X}(x) \pi_{B}(b)$ for $a \in A, x \in X, b \in B$.

Remarks. (1) The representations of ${ }_{A} X_{B}$ on the Hilbert spaces $(\mathscr{H}, \mathscr{K})$ are the nondegenerate imprimitivity-bimodule homomorphisms of $X$ into $M(K(\mathscr{K}, \mathscr{H}))$, where $K(\mathscr{K}, \mathscr{H})$ is viewed as a $K(\mathscr{H})-K(\mathscr{K})$ imprimitivity bimodule.

(2) Equation (1) together with the equation $\|T\|^{2}=\left\|T^{*} T\right\|$ for $T \in B(\mathscr{K}, \mathscr{H})$ implies that $\pi_{X}$ is isometric iff either $\pi_{A}$ or $\pi_{B}$ is isometric, and hence iff either $\pi_{A}$ or $\pi_{B}$ is faithful. If so, we say that $\pi$ is faithful.

(3) The map $\pi_{X}$ is automatically nondegenerate: $\operatorname{sp}\left\{\pi_{X}(x) k: x \in X, k \in \mathscr{K}\right\}$ is dense in $\mathscr{H}$ and $\operatorname{sp}\left\{\pi_{X}(x)^{*} h: x \in X, h \in \mathscr{H}\right\}$ is dense in $\mathscr{K}$. For if $h \in \mathscr{H}$ satisfies $\left(\pi_{X}(x) k \mid h\right)=0$ for all $x, k$, then

$$
\left(\pi_{A}\left({ }_{A}\langle x, y\rangle\right) g \mid h\right)=\left(\pi_{X}(x)\left(\pi_{X}(y)^{*} g\right) \mid h\right)=0
$$

for all $x, y \in X$ and $g \in \mathscr{H}$, which implies $h=0$ by nondegeneracy of $\pi_{A}$. Thus $\overline{\mathrm{sp}}\left\{\pi_{X}(x) k: x \in X, k \in \mathscr{K}\right\}=\mathscr{H}$; the other part follows similarly from the nondegeneracy of $\pi_{B}$. 
(4) We say two representations $\left(\pi_{A}, \pi_{X}, \pi_{B}\right),\left(\rho_{A}, \rho_{X}, \rho_{B}\right)$ on $\left(\mathscr{H}_{\pi}, \mathscr{K}_{\pi}\right),\left(\mathscr{H}_{\rho}, \mathscr{K}_{\rho}\right)$ are equivalent if there are unitary transformations $U: \mathscr{H}_{\pi} \rightarrow \mathscr{H}_{\rho}, V: \mathscr{K}_{\pi} \rightarrow \mathscr{K}_{\rho}$ such that $\rho_{X}(x)=U \pi_{X}(x) V^{*}$. It follows from equation (2) that we then have $\rho_{A}=\operatorname{Ad} U \circ \pi_{A}, \rho_{B}=\operatorname{Ad} V \circ \pi_{B}$.

(5) If $\left(\pi_{A}, \pi_{X}, \pi_{B}\right)$ is a representation of ${ }_{A} X_{B}$ on $(\mathscr{H}, \mathscr{K})$, and we set $\pi_{\tilde{X}}(\tilde{x}):=$ $\pi_{X}(x)^{*}$, then $\left(\pi_{B}, \pi_{\tilde{X}}, \pi_{A}\right)$ is a representation of ${ }_{B} \tilde{X}_{A}$. Thus if $\pi$ is faithful, and we use $\pi_{A}, \pi_{B}$ to view $A, B$ as acting spatially on $\mathscr{H}, \mathscr{K}$, and $\pi_{X}$ to view $X$ as a subspace of $B(\mathscr{K}, \mathscr{H})$, then we can identify $\tilde{X}$ with the subspace $X^{*}$ of $B(\mathscr{H}, \mathscr{K})$, with the module actions given by composition of operators, and (e.g.) ${ }_{B}\left\langle x^{*}, y^{*}\right\rangle=\langle x, y\rangle_{B}$.

(6) If ${ }_{A} X_{B}$ is represented on $(\mathscr{H}, \mathscr{K})$ via $\pi=\left(\pi_{A}, \pi_{X}, \pi_{B}\right),{ }_{B} Y_{C}$ is represented on $(\mathscr{K}, \mathscr{L})$ via $\rho=\left(\rho_{B}, \rho_{Y}, \rho_{C}\right)$, and $\pi_{B}=\rho_{B}$, then ${ }_{A}\left(X \otimes_{B} Y\right)_{C}$ is represented on $(\mathscr{H}, \mathscr{L})$ via $\sigma=\left(\pi_{A}, \sigma_{X \otimes_{B} Y}, \rho_{C}\right)$ where $\sigma_{X \otimes_{B} Y}(x \otimes y)=\pi_{X}(x) \rho_{Y}(y) \in B(\mathscr{L}, \mathscr{H})$. If $\pi$ or $\rho$ is faithful, then Remark (2) implies that $\sigma$ is also faithful.

LEMmA 2.2. The map $\left(\pi_{A}, \pi_{X}, \pi_{B}\right) \mapsto \pi_{B}$ is a bijection between the equivalence classes of representations of the $A-B$ imprimitivity bimodule $X$ and the equivalence classes of nondegenerate representations of $B$, with inverse given by $\pi \mapsto$ (Ind $\pi, \rho, \pi$ ), where Ind $\pi$ denotes the induced representation of $A$ on $X \otimes_{B} \mathscr{K}$ via the action of $A$ on $X$, and $\rho(x) k=x \otimes k \in X \otimes_{B} \mathscr{K}$.

Proof. We first claim that (Ind $\pi, \rho, \pi)$ is a representation of $X$. For $k \in \mathscr{K}$, we have

$$
\begin{aligned}
\rho(a \cdot x \cdot b) k & =a \cdot x \cdot b \otimes k=(a \cdot x) \otimes \pi(b) k \\
& =\operatorname{Ind} \pi(a)(x \otimes \pi(b) k)=\operatorname{Ind} \pi(a) \rho(x) \pi(b) k,
\end{aligned}
$$

which gives (1), and the definition of the inner product on $X \otimes_{B} \mathscr{K}$ implies immediately that $\rho(x)^{*} \rho(y)=\pi\left(\langle x, y\rangle_{B}\right)$. A quick calculation shows that $\rho(y)^{*}(z \otimes k)=\pi\left(\langle y, z\rangle_{B}\right) k$, so

$$
\begin{aligned}
\rho(x) \rho(y)^{*}(z \otimes k) & =x \otimes \pi\left(\langle y, z\rangle_{B}\right) k=\left(x \cdot\langle y, z\rangle_{B}\right) \otimes k \\
& =\left({ }_{A}\langle x, y\rangle \cdot z\right) \otimes k=\operatorname{Ind} \pi\left({ }_{A}\langle x, y\rangle\right)(z \otimes k),
\end{aligned}
$$

completing the proof of (2). It is easy to check that $\pi \rightarrow($ Ind $\pi, \rho, \pi)$ respects unitary equivalence, and $\left(\pi_{A}, \pi_{X}, \pi_{B}\right) \mapsto \pi_{B}$ certainly does, so it remains to check that $\left(\pi_{A}, \pi_{X}, \pi_{B}\right) \sim\left(\right.$ Ind $\left.\pi_{B}, \rho, \pi_{B}\right)$. The formula $U(x \otimes k):=\pi_{X}(x) k$ defines an isometry $U$ of $X \otimes_{B} \mathscr{K}$ into $\mathscr{H}$, which is surjective by Remark (3) above, and hence unitary. Straightforward calculations show that $U$ Ind $\pi_{B}=\pi_{A} U$ and $U \rho=\pi_{X}$, so $(U, 1)$ implements the required equivalence between $\left(\pi_{A}, \pi_{X}, \pi_{B}\right)$ and (Ind $\pi_{B}, \rho, \pi_{B}$ ).

COROLlARY 2.3. Every imprimitivity bimodule has a faithful representation.

Proof. If $\pi_{B}$ is a faithful representation of $B$, then each of $\pi_{A}$ and $\pi_{X}$ is faithful by Remark (2) above. So the result follows from the lemma. 
Proposition 2.4. Let $\pi=\left(\pi_{A}, \pi_{X}, \pi_{B}\right)$ be a representation of an imprimitivity bimodule ${ }_{A} X_{B}$ on $(\mathscr{H}, \mathscr{K})$. Then there is a unique strict-to-*-strong continuous map $\bar{\pi}_{X}: M(X) \rightarrow B(\mathscr{K}, \mathscr{H})$ which extends $\pi_{X}$, and is compatible with the canonical extensions of $\pi_{A}, \pi_{B}$ to $M(A)$ and $M(B)$, respectively. If $\pi$ is faithful, then $\pi_{X}$ is an isometry of $M(X)$ onto

$$
M_{\pi}(X)=\left\{T \in B(\mathscr{K}, \mathscr{H}): T \pi_{B}(B) \cup \pi_{A}(A) T \subseteq \pi_{X}(X)\right\} .
$$

Proof. We define $\bar{\pi}_{X}(m)$ by $\bar{\pi}_{X}(m)\left(\pi_{A}(b) k\right)=\pi_{X}(m \cdot b) k$; the argument used in Proposition 1.9 shows that $\bar{\pi}_{X}(m)$ is a well defined linear map. Easy calculations show that $\bar{\pi}_{X}(m)^{*}\left(\pi_{A}(a) h\right)=\pi_{X}(a \cdot m)^{*} h$ defines an adjoint for $\bar{\pi}_{X}(m)$. It is clear that $\bar{\pi}_{X}$ is strictly continuous with range in $M_{\pi}(X)$.

If $\pi_{B}$ is faithful, then $\bar{\pi}_{B}$ is isometric, and

$$
\left\|\bar{\pi}_{X}(m)\right\|^{2}=\left\|\bar{\pi}_{X}(m)^{*} \bar{\pi}_{X}(m)\right\|=\left\|\bar{\pi}_{B}\left(\langle m, m\rangle_{M(B)}\right)\right\|=\left\|\langle m, m\rangle_{M(B)}\right\| .
$$

Conversely, because $\pi_{X}$ is bijective, any $T$ in $M_{\pi}(X)$ defines a pair of maps $\left(n_{A}, n_{B}\right)$ via

$$
\pi_{X}\left(n_{A}(a)\right)=\pi_{A}(a) T, \pi_{X}\left(n_{B}(b)\right)=T \pi_{B}(b),
$$

and $n=\left(n_{A}, n_{B}\right)$ is easily seen to be a multiplier $n$ of $X$ satisfying $\bar{\pi}_{X}(n)=T$.

Note that the extension $\bar{\pi}$ in the proposition is identical to the unique strictly continuous extension of $\pi$ regarded as an imprimitivity bimodule homomorphism from ${ }_{A} X_{B}$ to $M\left({ }_{K(\mathscr{H})} K(\mathscr{K}, \mathscr{H})_{K(\mathscr{K})}\right)$ (see Proposition 1.9).

ExAMPLE 2.5. If $\mathscr{H}, \mathscr{K}$ are two Hilbert spaces then $X=K(\mathscr{K}, \mathscr{H})$ is a $K(\mathscr{H})-K(\mathscr{K})$ imprimitivity bimodule. Of course, the identity id is a faithful representation of $X$ on $(\mathscr{H}, \mathscr{K})$, so Proposition 2.4 gives an isomorphism of $M(X)$ onto $M_{\mathrm{id}}(X)$, which in this case is all of $B(\mathscr{K}, \mathscr{H})$. Hence $M(X)=B(\mathscr{K}, \mathscr{H})$. If one of $\mathscr{H}, \mathscr{K}$ is finite dimensional, then we have $M(X)=X$, which then implies that the ranges $R(A)$ and $R(B)$ of the inner products on $M(X)$ (see the remark following Proposition 1.6) are equal to $A=K(\mathscr{H})$ and $B=K(\mathscr{K})$, respectively. If $\mathscr{H}, \mathscr{K}$ have the same infinite dimension, then $M(X)=B(\mathscr{K}, \mathscr{H}), R(A)=B(\mathscr{H})$ and $R(B)=B(\mathscr{K})$. Thus $M(X)$ is a $B(\mathscr{H})-B(\mathscr{K})$ imprimitivity bimodule. But if $\mathscr{H}, \mathscr{K}$ have different infinite dimensions - say $\mathscr{H}$ is countable - then $R(A)$ is the ideal of $B(\mathscr{H})=M(K(\mathscr{H}))$ consisting of all operators $T$ such that range $T$ is separable. So in this case we have $A \neq \mathrm{R}(\mathrm{A}) \neq M(A)$, but $R(B)=M(B)$.

The following lemma will be needed in the next section. All our tensor products of $C^{*}$-algebras are completed with respect to the minimal (or spatial) norm.

LEMMA 2.6. Let $\pi=\left(\pi_{A}, \pi_{X}, \pi_{B}\right)$ be a faithful representation of ${ }_{A} X_{B}$ on Hilbert spaces $(\mathscr{H}, \mathscr{K})$, and let $\rho$ be a faithful representation of a $C^{*}$-algebra $C$ on the Hilbert space $\mathscr{L}$. Then there is a faithful representation $\pi \otimes \rho=\left(\pi_{A} \otimes \rho, \pi_{X} \otimes \rho\right.$, 
$\left.\pi_{\mathrm{B}} \otimes \rho\right)$ of ${ }_{A \otimes C}(X \otimes C)_{B \otimes C}$ on $(\mathscr{H} \otimes \mathscr{L}, \mathscr{K} \otimes \mathscr{L})$, such that $\pi_{A} \otimes \rho$ and $\pi_{B} \otimes \delta$ are the usual tensor product representations, and $\pi_{X} \otimes \rho(x \otimes c)=\pi_{X}(x) \otimes \rho(c)$.

Proof. For any $x \in X, c \in C$, there is a bounded operator $\pi_{X}(x) \otimes \rho(c)$ : $\mathscr{K} \otimes \mathscr{L} \rightarrow \mathscr{H} \otimes \mathscr{L}$, and hence a linear map $\pi_{X} \otimes \rho: X \odot C \rightarrow B(\mathscr{K} \otimes \mathscr{L}$, $\mathscr{H} \otimes \mathscr{L})$. A calculation using the equality $\pi_{B}\left(\langle x, y\rangle_{B}\right)=\pi_{X}(x)^{*} \pi_{X}(y)$ shows that $\left\|\pi_{X} \otimes \rho\left(\Sigma_{i=1}^{m} x_{i} \otimes c_{i}\right)\right\|^{2}=\left\|\Sigma_{i=1}^{m} x_{i} \otimes c_{i}\right\|_{B \otimes C}^{2}$, and hence $\pi_{X} \otimes \rho$ extends to an isometric linear map of $X \otimes C$ into $B(\mathscr{K} \otimes \mathscr{L}, \mathscr{H} \otimes \mathscr{L})$. Calculations on elementary tensors show that $\pi_{X} \otimes \rho$ is compatible with $\pi_{A} \otimes \rho$ and $\pi_{B} \otimes \rho$.

\section{§3. Morita equivalent coactions.}

We now give our versions of the theorems of Baaj-Skandalis and Bui. Throughout we shall use the conventions of $[7,10]$ as regards coactions: thus we use the minimal tensor product and the reduced group $C^{*}$-algebra. The comultiplication $\delta_{G}$ on $C_{r}^{*}(G)$ is the integrated form of the unitary representation $s \mapsto \lambda_{s}^{G} \otimes \lambda_{s}^{G}$ of $G$, where $\lambda^{G}$ denotes the left regular representation of $G$ on $L^{2}(G)$. In the following $\iota$ always denotes the identity homomorphism between algebras, and $M$ the representation of $C_{0}(G)$ as multiplication operators on $L^{2}(G)$. We shall find it convenient to distinguish between the function $w_{G}: s \rightarrow \lambda_{s}^{G}$ in $\left.M\left(G, C_{r}^{*}(G)\right)\right)$ and the unitary operator $W_{G}=M \otimes \imath\left(w_{G}\right)$ on $L^{2}(G \times G)$.

Definition 3.1 (cf. $[1,2.2],[3,2.15])$. Suppose that $\delta_{A}: A \rightarrow M\left(A \otimes C_{r}^{*}(G)\right)$ and $\delta_{B}: B \rightarrow M\left(B \otimes C_{r}^{*}(G)\right)$ are coactions of a locally compact group $G$ on $C^{*}$-algebras $A$ and $B$. A Morita equivalence between $\left(A, \delta_{A}\right)$ and $\left(B, \delta_{B}\right)$ is an imprimitivity bimodule ${ }_{A} X_{B}$ together with a linear map $\delta_{X}: X \rightarrow M\left(X \otimes C_{r}^{*}(G)\right)$ satisfying

(1) $\left(1_{M(A)} \otimes z\right) \cdot \delta_{X}(x)$ and $\delta_{X}(x) \cdot\left(1_{M(B)} \otimes z\right)$ lie in $X \otimes C_{r}^{*}(G)$ for all $x \in X$, $z \in C_{r}^{*}(G)$

(2) $\delta_{X}(a \cdot x \cdot b)=\delta_{A}(a) \cdot \delta_{X}(x) \cdot \delta_{B}(b)$ for all $a \in A, x \in X, b \in B$;

(3) $\delta_{A}\left({ }_{A}\langle x, y\rangle\right)={ }_{M\left(A \otimes C_{r}^{*}(G)\right)}\left\langle\delta_{X}(x), \delta_{X}(y)\right\rangle$, and $\delta_{B}\left(\langle x, y\rangle_{B}\right)=\left\langle\delta_{X}(x), \delta_{X}(y)\right\rangle_{M\left(B \otimes C_{r}^{*}(G)\right)}$ for all $x, y \in X$;

(4) $\left(\delta_{X} \otimes \imath\right) \circ \delta_{X}=\left(\imath \otimes \delta_{G}\right) \circ \delta_{X}$.

(In (1) and (2), we implicitly extended the module actions on the $A \otimes C_{r}^{*}(G)-$ $B \otimes C_{r}^{*}(G)$ imprimitivity bimodule $X \otimes C_{r}^{*}(G)$ to actions of the multiplier algebras on the multiplier bimodule; in (3), we extended the inner products to $M\left(X \otimes C_{r}^{*}(G)\right)$; and in (4), we used the strictly continuous extensions of $\delta_{X} \otimes \imath$ and $\imath \otimes \delta_{G}$ to make sense of the compositions.)

Remarks. We claim that this version of Morita equivalence is indeed an equivalence relation. Obviously, $\left(A, \delta_{A}\right)$ is equivalent to itself: take $\left(X, \delta_{X}\right)=$ $\left(A, \delta_{A}\right)$. If $\left(X, \delta_{X}\right)$ is an equivalence between $\left(A, \delta_{A}\right)$ and $\left(B, \delta_{B}\right)$, we can use a faithful 
representation of ${ }_{A} X_{B}$ and part (5) of the remark preceding Lemma 2.2 above to identify ${ }_{B} \tilde{X}_{A}$ with $X^{*}$. Then, if we represent $X \otimes C_{r}^{*}(G)$ faithfully on $B\left(\mathscr{K} \otimes L^{2}(G), \mathscr{H} \otimes L^{2}(G)\right)$ using Lemma 2.6,

$$
\tilde{X} \otimes C_{r}^{*}(G)=X^{*} \otimes C_{r}^{*}(G)=\left(X \otimes C_{r}^{*}(G)\right)^{*},
$$

and we can define $\delta_{\tilde{X}}: \tilde{X} \rightarrow M\left(\tilde{X} \otimes C_{r}^{*}(G)\right)$ by $\delta_{\tilde{X}}\left(x^{*}\right)=\delta_{X}(x)^{*}$. It is routine to check that $\left(\tilde{X}, \delta_{\tilde{X}}\right)$ is an equivalence between $\left(B, \delta_{B}\right)$ and $\left(A, \delta_{A}\right)$. To see transitivity, suppose that $\left({ }_{A} X_{B}, \delta_{X}\right)$ and $\left({ }_{B} Y_{C}, \delta_{Y}\right)$ are Morita equivalences. We represent ${ }_{B} Y_{C}$ in $B(\mathscr{L}, \mathscr{K})$, and then use Lemma 2.2 to represent ${ }_{A} X_{B}$ in $B(\mathscr{K}, \mathscr{H})$ for the same space $\mathscr{K}$. Now $X \otimes_{B} Y$ is represented in $B(\mathscr{L}, \mathscr{H})$, and $\left(X \otimes_{B} Y\right) \otimes C_{r}^{*}(G)$ in $B\left(\mathscr{L} \otimes L^{2}(G), \mathscr{H} \otimes L^{2}(G)\right)$. We define

$$
\delta_{X \otimes_{B} Y}(x \otimes y):=\delta_{X}(x) \circ \delta_{Y}(y) .
$$

Routine calculations, using the realisations on Hilbert space, show that $\delta_{X \otimes_{B} Y}$ satisfies (1) and (2). For (3), we have

$$
\begin{aligned}
M\left(A \otimes C_{r}^{*}(G)\right) & \left\langle\delta_{X}(x) \delta_{Y}(y), \delta_{X}(z) \delta_{Y}(w)\right\rangle=\delta_{X}(x) \delta_{Y}(y) \delta_{Y}(w)^{*} \delta_{X}(z)^{*} \\
& =\delta_{X}(x) \delta_{B}\left(\langle y, w\rangle_{B}\right) \delta_{X}(z)^{*}=\delta_{X}(x)\left(\delta_{X}(z) \delta_{B}\left({ }_{B}\langle w, y\rangle\right)\right)^{*} \\
& =\delta_{X}(x) \delta_{X}\left(z \cdot{ }_{B}\langle w, y\rangle\right)^{*}=\delta_{A}\left({ }_{A}\left\langle x, z \cdot{ }_{B}\langle w, y\rangle\right\rangle\right) \\
& =\delta_{A}\left({ }_{A}\langle x \otimes y, z \otimes w\rangle\right) .
\end{aligned}
$$

Finally, we check (4). Since the faithful representation of $X \otimes_{B} Y$ in $B(\mathscr{L}, \mathscr{H})$ takes $x \otimes y$ to $x \circ y$, we have

$$
\begin{aligned}
& \left(\delta_{X} \otimes_{B} Y \otimes l\right)\left(\delta_{X}(x) \delta_{Y}(y)\right)=\left(\delta_{X} \otimes l\right)\left(\delta_{X}(x)\right) \circ\left(\delta_{Y} \otimes \imath\right)\left(\delta_{Y}(y)\right) \\
& \quad=\left(l \otimes \delta_{G}\right)\left(\delta_{X}(x)\right) \circ\left(l \otimes \delta_{G}\right)\left(\delta_{Y}(y)\right)=\left(l \otimes \delta_{G}\right)\left(\delta_{X}(x) \delta_{Y}(y)\right) \\
& \quad=\left(l \otimes \delta_{G}\right)\left(\delta_{X \otimes_{B} Y}(x \otimes y)\right) .
\end{aligned}
$$

From now on, we shall represent the imprimitivity bimodule ${ }_{A} X_{B}$ faithfully on $(\mathscr{H}, \mathscr{K})$ and ${ }_{A \otimes C_{r}^{*}(G)}\left(X \otimes C_{r}^{*}(G)\right)_{B \otimes C_{r}^{*}(G)}$ on $\left(\mathscr{H} \otimes L^{2}(G), \mathscr{K} \otimes L^{2}(G)\right)$ using Lemma 2.6. Thus we may identify the multiplier algebras $M\left(A \otimes C_{r}^{*}(G)\right), M\left(B \otimes C_{r}^{*}(G)\right)$ and the multiplier bimodule $M\left(X \otimes C_{r}^{*}(G)\right)$ with their images in $B\left(\mathscr{H} \otimes L^{2}(G)\right)$, $B\left(\mathscr{K} \otimes L^{2}(G)\right)$ and $B\left(\mathscr{K} \otimes L^{2}(G), \mathscr{H} \otimes L^{2}(G)\right)$, respectively. If $\delta_{A}$ and $\delta_{B}$ are coactions of $G$ on $A$ and $B$, respectively, then the crossed products $A \times_{\delta_{A}} G$ and $B \times{ }_{\delta_{B}} G$ can be realized in $B\left(\mathscr{H} \otimes L^{2}(G)\right)$ and $B\left(\mathscr{K} \otimes L^{2}(G)\right)$ as the closed linear spans of $\left\{\delta_{A}(a)\left(1 \otimes M_{f}\right) ; a \in A, f \in C_{0}(G)\right\}$ and $\left\{\delta_{B}(b)\left(1 \otimes M_{f}\right) ; b \in B, f \in C_{0}(G)\right\}$, respectively [7].

THEOREM 3.2 (Baaj-Skandalis, see $[1,6.9]$, [3,2.16]). Suppose $\left({ }_{A} X_{B}, \delta_{X}\right)$ is 
a Morita equivalence between $\left(A, \delta_{A}\right)$ and $\left(B, \delta_{B}\right)$ such that ${ }_{A} X_{B}$ is faithfully represented on $(\mathscr{H}, \mathscr{K})$. Then the subspace

$$
X \times_{\delta_{X}} G:=\overline{\operatorname{sp}}\left\{\delta_{X}(x)\left(1 \otimes M_{f}\right): x \in X, f \in C_{0}(G)\right\}
$$

of $B\left(\mathscr{K} \otimes L^{2}(G), \mathscr{H} \otimes L^{2}(G)\right)$ is an $A \times_{\delta_{A}} G-B \times{ }_{\delta_{B}} G$ imprimitivity bimodule.

Indeed,

$$
X \times_{\delta_{X}} G=\overline{\operatorname{sp}}\left\{\left(1 \otimes M_{f}\right) \delta_{X}(x): x \in X, f \in C_{0}(G)\right\},
$$

the algebras $A \times_{\delta_{A}} G$ act through their faithful representations on $\mathscr{H} \otimes L^{2}(G)$, $\mathscr{K} \otimes L^{2}(G)$, respectively, and the inner products are given in terms of the usual adjoint * : $B\left(\mathscr{K} \otimes L^{2}(G), \mathscr{H} \otimes L^{2}(G)\right) \rightarrow B\left(\mathscr{H} \otimes L^{2}(G), \mathscr{K} \otimes L^{2}(G)\right) b y$

$$
\langle\eta, \zeta\rangle_{B \times \delta_{B} G}=\eta^{*} \zeta,{ }_{A \times \delta_{A} G}\langle\eta, \zeta\rangle=\eta \zeta^{*} \text {. }
$$

Further, the maps $\phi: x \otimes c \mapsto \delta_{X}(x) \cdot c, \psi: d \otimes x \mapsto d \cdot \delta_{X}(x)$ extend to $B \times_{\delta_{B}} G-$ resp. $A \times_{\delta_{A}} G$-linear Hilbert module isomorphisms of $X \otimes_{B}\left(B \times_{\delta_{B}} G\right)$ onto $X \times_{\delta_{X}} G$ and $\left(A \times_{\delta_{A}} G\right) \otimes_{A} \times X$ onto $X \times_{\delta_{X}} G$, respectively.

Proof. We begin by proving that $\left(1 \otimes M_{f}\right) \delta_{X}(x)$ belongs to $X \times_{\delta_{X}} G$. The argument is basically that used to prove that $\left(1 \otimes M_{f}\right) \delta_{A}(a)$ belongs to $\overline{\mathrm{sp}}\left\{\delta_{A}(a)\left(1 \otimes M_{f}\right)\right\}$ in [7, p. 759], and this fact will also be used later in the proof. By continuity, we may as well suppose that $f \in A(G)$, the Fourier algebra of $G$, and indeed that the functional $f \in L(G)_{*}$ is given by $T \mapsto(z T h \mid k)$ for some $z \in C_{r}^{*}(G)$ and $h, k \in L^{2}(G)$ (in other words, if $g \in A(G)$ is given as a functional by $\langle T, g\rangle=(T h \mid k)$, then $f=g \cdot z)$. Since $f=S_{f}\left(W_{G}\right), M_{f}$ is characterised spatially by

$$
\left(M_{f} \xi \mid \eta\right)=\left((1 \otimes z) W_{G}(\xi \otimes h) \mid \eta \otimes k\right) \text { for } \xi, \eta \in L^{2}(G) .
$$

Notice that the equation $\delta_{G}(z)=W_{G}(z \otimes 1) W_{G}^{*}$ tensors up, first to $X \otimes C_{r}^{*}(G)$, and then by strict continuity to $M\left(X \otimes C_{r}^{*}(G)\right)$, to give $\left(l \otimes \delta_{G}\right) \circ \delta_{X}(x)=$ $\left(1 \otimes W_{G}\right)\left(\delta_{X}(x) \otimes 1\right)\left(1 \otimes W_{G}\right)^{*}$. Thus, for $\xi \in \mathscr{K} \otimes L^{2}(G)$ and $\eta \in \mathscr{H} \otimes L^{2}(G)$, we have

$$
\begin{aligned}
\left(\left(1 \otimes M_{f}\right) \delta_{X}(x) \xi \mid \eta\right) & =\left((1 \otimes 1 \otimes z)\left(1 \otimes W_{G}\right)\left(\delta_{X}(x) \otimes 1\right) \xi \otimes h \mid \eta \otimes k\right) \\
& =\left((1 \otimes 1 \otimes z)\left(\imath \otimes \delta_{G}\right) \circ \delta_{X}(x)\left(1 \otimes W_{G}\right) \xi \otimes h \mid \eta \otimes k\right) \\
& =\left((1 \otimes 1 \otimes z)\left(\delta_{X} \otimes \imath\right) \circ \delta_{X}(x)\left(1 \otimes W_{G}\right) \xi \otimes h \mid \eta \otimes k\right) \\
& \left.=\left(\delta_{X} \otimes \imath((1 \otimes z)) \delta_{X}(x)\right)\left(1 \otimes W_{G}\right) \xi \otimes h \mid \eta \otimes k\right) .
\end{aligned}
$$

Since $(1 \otimes z) \delta_{X}(x) \in X \otimes C_{r}^{*}(G)$, we can approximate it in norm by a sum of the form $\Sigma_{i} x_{i} \otimes z_{i}$ in $X \odot C_{r}^{*}(G)$. But then we have 


$$
\begin{aligned}
\left(\left(1 \otimes M_{f}\right) \delta_{X}(x) \xi \mid \eta\right) & \sim\left(\sum_{i}\left(\left(\delta_{X}\left(x_{i}\right) \otimes 1\right)\left(1 \otimes 1 \otimes z_{i}\right)\right)\left(1 \otimes W_{G}\right) \xi \otimes h \mid \eta \otimes k\right) \\
& =\left(\sum_{i} \delta_{X}\left(x_{i}\right)\left(1 \otimes M_{g \cdot z_{l}}\right) \xi \mid \eta\right),
\end{aligned}
$$

and the approximation is uniform in $\xi, \eta$ of norm $\leqq 1$. Thus

$$
\left(1 \otimes M_{f}\right) \delta_{X}(x) \sim \sum_{i} \delta_{X}\left(x_{i}\right)\left(1 \otimes M_{g \cdot z_{i}}\right) \in X \times_{\delta_{X}} G,
$$

as claimed.

By symmetry, this claim establishes (3.2) The claim also gives

$$
\delta_{A}(a)\left(1 \otimes M_{f}\right) \delta_{X}(x) \sim \delta_{A}(a) \sum_{i} \delta_{X}\left(x_{i}\right)\left(1 \otimes M_{g \cdot z_{\imath}}\right)=\sum_{i} \delta_{X}\left(a \cdot x_{i}\right)\left(1 \otimes M_{g \cdot z_{\imath}}\right) ;
$$

because the elements of the form $\delta_{A}(a)\left(1 \otimes M_{f}\right)$ span a dense subspace of $A \times_{\delta_{A}} G$, this implies that the subspace $X \times_{\delta_{X}} G$ of $B\left(\mathscr{K} \otimes L^{2}(G), \mathscr{H} \otimes L^{2}(G)\right)$ is closed under left multiplication by elements of $A \times_{\delta_{A}} G \subset B\left(\mathscr{H} \otimes L^{2}(G)\right)$. Similarly, the calculation in [LPRS, p. 759] implies that $X \times_{\delta_{X}} G$ is closed under right multiplication by elements of $B \times{ }_{\delta_{B}} G$. Thus $X \times{ }_{\delta_{X}} G$ is an $A \times \times_{\delta_{A}} G-B \times{ }_{\delta_{B}} G$ bimodule.

We next note that

$$
\begin{aligned}
\left(\delta_{X}(x)\left(1 \otimes M_{f}\right)\right) *\left(\delta_{X}(y)\left(1 \otimes M_{g}\right)\right) & =\left(1 \otimes M_{\bar{f}}\right)\left(\delta_{X}(x)^{*} \delta_{X}(y)\right)\left(1 \otimes M_{g}\right) \\
& =\left(1 \otimes M_{\bar{f}}\right) \delta_{B}\left(\langle x, y\rangle_{B}\right)\left(1 \otimes M_{g}\right),
\end{aligned}
$$

so $\eta^{*} \zeta$ in (3.3) does lie in $B \times_{\delta_{B}} G$. Since the pairing is defined in terms of the usual adjoint operation on Hilbert space, there is no difficulty in verifying the algebraic properties of $\langle\cdot, \cdot\rangle_{B \times \delta_{B} G}$, and since the elements of the form (3.5) span a dense subspace of $B \times \times_{\delta_{B}} G$, it follows that $X \times_{\delta_{B}} G$ is a full Hilbert $B \times \times_{\delta_{B}} G$ module. Similarly, using instead the characterisation (3.2) of $X \times_{\delta_{X}} G$, one can verify that it is a full Hilbert $A \times{ }_{\delta_{A}} G$ module. The compatibility condition

$$
{ }_{A} \times \delta_{A} G\langle\xi, \eta\rangle \cdot \zeta=\xi \cdot\langle\eta, \zeta\rangle_{B \times \delta_{B} G}
$$

amounts to the associativity of composition of operators, and hence $X \times_{\delta_{X}} G$ is an imprimitivity bimodule, as asserted.

For the last part, we observe that the map $\phi$ is clearly a $B \times_{\delta_{B}} G$ module homomorphism with dense range, and hence it is enough to check that $\phi$ is isometric for the $B \times_{\delta_{B}} G$ norm on $X \odot\left(B \times_{\delta_{B}} G\right)$ and the operator norm on $B\left(\mathscr{K} \otimes L^{2}(G), \mathscr{H} \otimes L^{2}(G)\right)$. But for $\Sigma_{i} x_{i} \otimes c_{i} \in X \odot\left(B \times_{\delta_{B}} G\right)$ we have 


$$
\begin{aligned}
\left\|\phi\left(\sum_{i} x_{i} \otimes c_{i}\right)\right\|^{2} & =\left\|\sum_{i, j} c_{i}^{*} \delta_{X}\left(x_{i}\right)^{*} \delta_{X}\left(x_{j}\right) c_{j}\right\| \\
& =\left\|\sum_{i, j} c_{i}^{*}\left\langle\delta_{X}\left(x_{i}\right), \delta_{X}\left(x_{j}\right)\right\rangle_{M\left(B \otimes c_{r}^{*}(G)\right) C_{j}}\right\| \\
& =\left\|\sum_{i, j} c_{i}^{*} \delta_{B}\left(\left\langle x_{i}, x_{j}\right\rangle_{B}\right) c_{j}\right\| \\
& =\left\|\sum_{i, j}\left\langle x_{i} \otimes c_{i}, x_{j} \otimes c_{j}\right\rangle_{B \times \delta_{B} G}\right\| \\
& =\left\|\sum_{i, j} x_{i} \otimes c_{i}\right\|_{X \otimes B_{B}\left(B \times \delta_{B} G\right)}^{2}
\end{aligned}
$$

Exactly the same argument, but using (3.2) instead of (3.1), shows that $\psi$ is an isomorphism.

Now suppose $N$ is a closed normal amenable subgroup of $G$, and let $q$ denote the quotient map of $C_{r}^{*}(G)$ onto $C_{r}^{*}(G / N)$, so that $q\left(\lambda_{s}^{G}\right)=\lambda_{s N}^{G / N}$ for $s \in G$. For groups $H, K$, let $\sigma_{H, K}: C_{r}^{*}(H) \otimes C_{r}^{*}(K) \rightarrow C_{r}^{*}(K) \otimes C_{r}^{*}(H)$ denote the flip $w \otimes$ $z \mapsto z \otimes w$. As in [10], a twisted coaction of $(G, N)$ on $A$ consists of a pair $\left(\delta_{A}, W_{A}\right)$, where $\delta_{A}$ is a coaction of $G$ on $A$ and $W_{A}$ is a unitary in $M\left(A \otimes C_{r}^{*}(G / N)\right)$ satisfying

(1) $\left(W_{A} \otimes 1\right) l \otimes \sigma_{G / N, G / N}\left(W_{A} \otimes 1\right)=\left(l \otimes \delta_{G / N}\right)\left(W_{A}\right)$

(2) $(l \otimes q) \circ \delta_{A}(a)=W_{A}(a \otimes 1) W_{A}^{*}$ for all $a \in A$; and

(3) $\delta_{A} \otimes \imath\left(W_{A}\right)=\imath \otimes \sigma_{G / N, G}\left(W_{A} \otimes 1_{C_{r}^{*}(G)}\right)$.

A covariant representation of $\left(A, G, \delta_{A}\right)$ is a pair $(\pi, \mu)$ of nondegenerate representations $\pi: A \rightarrow B(\mathscr{H}), \mu: C_{0}(G) \rightarrow B(\mathscr{H})$ such that

$$
\pi \otimes \imath\left(\delta_{A}(a)\right)=\mu \otimes \imath\left(w_{G}\right)(\pi(a) \otimes 1) \mu \otimes \imath\left(w_{G}^{*}\right)
$$

and $(\pi, \mu)$ preserves the twist $W_{A}$ if

$$
\mu \otimes \imath\left(w_{G / N}\right)=\pi \otimes l\left(W_{A}\right) .
$$

The twisted crossed product $A \times_{\delta_{A}, W_{A}} G$ is then the quotient of $A \times_{\delta_{A}} G$ by the ideal

$$
I_{W_{A}}:=\cap\left\{\operatorname{ker} \pi \times \mu:(\pi, \mu) \text { is covariant and preserves } W_{A}\right\} .
$$

Following Bui [3], two twisted coactions $\left(\delta_{A}, W_{A}\right)$ and $\left(\delta_{B}, W_{B}\right)$ of $(G, N)$ are Morita equivalent if there exists a Morita equivalence $\left(X, \delta_{X}\right)$ for $\left(A, \delta_{A}\right)$ and $\left(B, \delta_{B}\right)$ such that 
$l \otimes q\left(\delta_{X}(x)\right)=W_{A} \cdot(x \otimes 1) \cdot W_{B}^{*}$ for all $x \in X$.

Using faithful representations of $X \otimes C_{r}^{*}(G)$ and $X \otimes C_{r}^{*}(G / N)$ on $\left(\mathscr{H} \otimes L^{2}(G)\right.$, $\left.\mathscr{K} \otimes L^{2}(G)\right)$ and $\left(\mathscr{H} \otimes L^{2}(G / N), \mathscr{K} \otimes L^{2}(G / N)\right)$, one can check that Morita equivalence of twisted actions is an equivalence relation (cf. the remarks following [3, Definition 3.2] and Definition 3.1).

Corollary 3.3 (Bui, cf. $[3,3.3])$. Suppose that $\left(X, \delta_{X}\right)$ is a Morita equivalence between the twisted systems $\left(A, \delta_{A}, W_{A}\right)$ and $\left(B, \delta_{B}, W_{B}\right)$. Then there is a submodule $E$ of $X \times_{\delta_{X}} G$ such that $\left(X \times_{\delta_{X}} G\right) / E$ is an $A \times_{\delta_{A}, W_{A}} G-B \times \times_{\delta_{B}, W_{B}} G$ imprimitivity bimodule.

Proof. We show that a covariant representation $(\pi, \mu)$ of $\left(B, \delta_{B}\right)$ preserves the twist $W_{B}$ if and only if the corresponding induced representation $(\tau, v)$ of $\left(A, \delta_{A}\right)$ preserves $W_{A}$-indeed, by symmetry, it is enough to show that if $(\pi, \mu)$ preserves $W_{B}$, then $(\tau, v)$ preserves $W_{A}$. This implies that the ideals $I_{W_{B}}$ and $I_{W_{A}}$ are in Rieffel correspondence, so that we can take $E=I_{W_{A}} \cdot\left(X \times_{\delta_{X}} G\right)=\left(X \times_{\delta_{X}} G\right) \cdot I_{W_{B}}$ (cf. [Rief2, §3]). We write $X \times_{\delta_{X}} G=\overline{\mathrm{sp}}\left\{\delta_{X}(x)\left(1 \otimes M_{f}\right)\right\}$, and understand that the closure is in some Hilbert space realisation like that in Theorem 3.2.

Suppose that $(\pi, \mu):\left(B, \delta_{B}\right) \rightarrow B(\mathscr{H})$ preserves $W_{B}$ and write $Y:=X \times_{\delta_{X}} G$. The induced representation $(\tau, v)$ acts in $Y \otimes_{B \times \delta_{B} G} \mathscr{H}$ via

$$
\begin{aligned}
& \tau(a)\left(\delta_{X}(x)\left(1 \otimes M_{f}\right) \otimes \xi\right)=\delta_{X}(a \cdot x)\left(1 \otimes M_{f}\right) \otimes \xi \\
& v(g)\left(\delta_{X}(x)\left(1 \otimes M_{f}\right) \otimes \xi\right)=\left(1 \otimes M_{g}\right) \delta_{X}(x)\left(1 \otimes M_{f}\right) \otimes \xi,
\end{aligned}
$$

and the key identity is

$$
y \cdot\left(\delta_{B}(b)\left(1 \otimes M_{f}\right)\right) \otimes \xi=y \otimes \pi(b) \mu(f) \xi
$$

for $y \in Y$. To calculate, we use the isomorphism

$\left(Y \otimes_{B \times \delta_{B} G} \mathscr{H}\right) \otimes L^{2}(G / N)=\left(Y \otimes C_{r}^{*}(G / N)\right) \otimes_{\left(B \times \delta_{B} G\right) \otimes C_{r}^{*}(G / N)}\left(\mathscr{H} \otimes L^{2}(G / N)\right)$.

Then for $\gamma:=\left(\delta_{X}(x)\left(1 \otimes M_{f}\right) \otimes \xi\right) \otimes z k$ in $(Y \otimes \mathscr{H}) \otimes L^{2}(G / N)$, we have $\tau \otimes l\left(W_{A}\right)\left(\delta_{X}(x)\left(1 \otimes M_{f}\right) \otimes \xi\right) \otimes z k$

$$
\begin{aligned}
& =\tau \otimes \imath\left(W_{A}\right)\left(\delta_{X}(x)\left(1 \otimes M_{f}\right) \otimes z\right) \otimes(\xi \otimes k) \\
& =\delta_{X} \otimes \imath\left(W_{A} \cdot(x \otimes 1)\right)\left(1 \otimes M_{f} \otimes z\right) \otimes(\xi \otimes k) \\
& =\delta_{X} \otimes \imath\left((\imath \otimes q) \circ \delta_{X}(x) \cdot W_{B}\right)\left(1 \otimes M_{f} \otimes z\right) \otimes(\xi \otimes k) \\
& =\imath \otimes \imath \otimes q\left(\left(\delta_{X} \otimes \imath\right) \circ \delta_{X}(x)\right) \delta_{B} \otimes \imath\left(W_{B}\right)\left(1 \otimes M_{f} \otimes z\right) \otimes(\xi \otimes k) \\
& =\imath \otimes \imath \otimes q\left(\left(\imath \otimes \delta_{G}\right) \circ \delta_{X}(x)\right) \delta_{B} \otimes \imath\left(W_{B}\right)\left(1 \otimes M_{f} \otimes z\right) \otimes(\xi \otimes k) \\
& =\imath \otimes \imath \otimes q\left(\left(1 \otimes W_{G}\right)\left(\delta_{X}(x) \otimes 1\right)\left(1 \otimes W_{G}^{*}\right)\right) \delta_{B} \otimes \imath\left(W_{B}\right)\left(1 \otimes M_{f} \otimes z\right) \otimes(\xi \otimes k) \\
& =\left(1 \otimes W_{G / N}\right)\left(\delta_{X}(x) \otimes 1\right)\left(1 \otimes W_{G / N}^{*}\right) \delta_{B} \otimes \imath\left(W_{B}\right)\left(1 \otimes M_{f} \otimes z\right) \otimes(\xi \otimes k) .
\end{aligned}
$$


At this stage, we observe that if $\zeta$ is any element of $Y \otimes C_{r}^{*}(G / N)$, then equations (3.6) and (3.7) give

$$
\begin{aligned}
\left(\zeta\left(1 \otimes W_{G / N}^{*}\right) \delta_{B} \otimes l\left(W_{B}\right)\right) & \left(1 \otimes M_{f} \otimes z\right) \otimes(\xi \otimes k) \\
= & \zeta \otimes\left(\mu \otimes l\left(w_{G / N}^{*}\right) \pi \otimes l\left(W_{B}\right)(\mu(f) \otimes z)(\xi \otimes k)\right) \\
= & \zeta \otimes(\mu(f) \otimes z)(\xi \otimes k) \\
= & \zeta\left(1 \otimes M_{f} \otimes z\right) \otimes(\xi \otimes k) .
\end{aligned}
$$

While this calculation does not immediately apply to our formula for $\tau \otimes l_{l}\left(W_{A}\right)(\gamma)$, because $\eta=\left(1 \otimes W_{G / N}\right)\left(\delta_{X}(x) \otimes 1\right)$ lies in $M\left(Y \otimes C_{r}^{*}(G / N)\right)$ rather than $Y \otimes C_{r}^{*}(G / N)$, one can see by considering $\left(\tau \otimes l\left(W_{A}\right)(\gamma) \mid \alpha\right)$ for $\alpha$ in $(Y \otimes$ $\left.C_{r}^{*}(G / N)\right) \otimes\left(\mathscr{H} \otimes L^{2}(G / N)\right)$ that the same manipulation works. (Since $C_{0}(G)$ acts nondegenerately on $Y$, we can take $\alpha$ of the form $\left(1 \otimes M_{g} \otimes w\right) \cdot \beta$, move $1 \otimes M_{g} \otimes w$ across the inner product, and note that $\zeta:=\left(1 \otimes M_{g} \otimes w\right)^{*} \eta$ does lie in $Y \otimes C_{r}^{*}(G / N)$.) We deduce that

$$
\begin{aligned}
\tau \otimes l\left(W_{A}\right)(\gamma) & =\left(\left(1 \otimes W_{G / N}\right)\left(\delta_{X}(x) \otimes 1\right)\left(1 \otimes M_{f} \otimes z\right)\right) \otimes(\xi \otimes k) \\
& =v \otimes \imath\left(w_{G / N}\right)\left(\left(\delta_{X}(x)\left(1 \otimes M_{f}\right) \otimes \xi\right) \otimes z k\right)
\end{aligned}
$$

and hence that $(\tau, v)$ preserves the twist $W_{A}$.

\section{Appendix: Linking algebras.}

In this appendix we shall relate the results of the first two sections to the linking algebra of an imprimitivity bimodule ${ }_{A} X_{B}$. To start with, recall that the adjoint imprimitivity bimodule $\tilde{X}$ is the set $X$ with left $B$-action and right $A$-action defined by $b \cdot \tilde{x}=\left(x \cdot b^{*}\right)$ and $\tilde{x} \cdot a=\left(a^{*} \cdot x\right) \tilde{)}$, where we write $\tilde{x}$ if we view $x \in X$ as an element of $\tilde{X}$. Equipped with the old $A$ - and $B$-valued inner products, $\tilde{X}$ is a $B$ - $A$ imprimitivity bimodule. Note that $M(\tilde{X})$ is naturally isomorphic to $M(X) \tilde{)}$. The linking algebra $L$ for ${ }_{A} X_{B}$ is $L=\left\{\left(\begin{array}{cc}a & x \\ \tilde{y} & b\end{array}\right): a \in A, x, y \in X, b \in B\right\}$, with multiplication and involution given by

$$
\left(\begin{array}{cc}
a_{1} & x_{1} \\
\tilde{y}_{1} & b_{1}
\end{array}\right)\left(\begin{array}{cc}
a_{2} & x_{2} \\
\tilde{y}_{2} & b_{2}
\end{array}\right)=\left(\begin{array}{cc}
a_{1} a_{2}+{ }_{A}\left\langle x_{1}, y_{2}\right\rangle & a_{1} \cdot x_{2}+x_{1} \cdot b_{2} \\
\tilde{y}_{1} \cdot a_{2}+b_{1} \cdot \tilde{y}_{2} & \left\langle y_{1}, x_{2}\right\rangle_{B}+b_{1} b_{2}
\end{array}\right)
$$

$$
\left(\begin{array}{ll}
a & x \\
\tilde{y} & b
\end{array}\right)^{*}=\left(\begin{array}{cc}
a^{*} & y \\
\tilde{x} & b^{*}
\end{array}\right)
$$

Each element of $L$ acts as an adjointable operator on $X \oplus B$ via

$$
\left(\begin{array}{ll}
a & x \\
\tilde{y} & b
\end{array}\right)\left(\begin{array}{l}
z \\
c
\end{array}\right)=\left(\begin{array}{c}
a \cdot z+x \cdot c \\
\langle y, z\rangle_{B}+b c
\end{array}\right)
$$


Indeed, it is shown in $[2, \S 1]$ that this action defines an isomorphism between $L$ and $\mathscr{K}_{B}(X \oplus B)$, and hence $L$ is a $C^{*}$-algebra. We shall often write $L=\left(\begin{array}{ll}A & X \\ \tilde{X} & B\end{array}\right)$.

Proposition A.1. Let $L=\left(\begin{array}{cc}A & X \\ \tilde{X} & B\end{array}\right)$ be the linking algebra for the imprimitivity bimodule ${ }_{A} X_{B}$. Then $M(L)$ can be naturally identified with $\left(\begin{array}{cc}M(A) & M(X) \\ M(\tilde{X}) & M(B)\end{array}\right)$, where we now use Proposition 1.6 to make sense of the formulas ( $A 1)$ for the multiplication and involution.

Proof. $L$ is an ideal in $\left(\begin{array}{cc}M(A) & M(X) \\ M(\tilde{X}) & M(B)\end{array}\right)$, because ${ }_{M(A)}\langle x, m\rangle \in A$ and $\langle x, m\rangle_{M(B)} \in$ $B$ for all $x \in X$ and $m \in M(X)$ (to see this, factor $x=a \cdot y$, and then $\langle a \cdot y, m\rangle_{M(B)}=$ $\left.\left\langle y, a^{*} \cdot m\right\rangle_{M(B)} \in B\right)$. Thus every element in $\left(\begin{array}{ll}M(A) & M(X) \\ M(\tilde{X}) & M(B)\end{array}\right)$ defines a multiplier of $L$. Conversely, let $p=p_{A}=\left(\begin{array}{cc}1_{A} & 0 \\ 0 & 0\end{array}\right)$ and $q=q_{B}=\left(\begin{array}{cc}0 & 0 \\ 0 & 1_{B}\end{array}\right)$. Then $p+q=1$ and we have canonical isomorphisms $A \cong p L p, B \cong q L q, X \cong p L q$ and $\tilde{X} \cong$ $q L p$. If $w \in M(L)$, then $p w p, p w q, q w p$, and $q w q$ define elements $k, m, \tilde{n}$ and $l$ in $M(A), M(X), M(\tilde{X})$, and $M(B)$ such that $w=\left(\begin{array}{cc}k & m \\ \tilde{n} & l\end{array}\right)$.

Remarks. (1) Proposition A.1 suggests that one could alternatively define the multiplier bimodule $M(X)$ to be the corner $p M(L) q$. However, that $M(X)$ then has the universal property of Proposition 1.2 is not immediately obvious, and a proof would involve much the same circle of ideas as Propositions 1.2 and 1.3.

(2) From the linking algebra point of view, the nondegenerate imprimitivitybimodule homomorphisms are in one-to-one correspondence with certain nondegenerate homomorphisms on $L=\left(\begin{array}{cc}A & X \\ \tilde{X} & B\end{array}\right)$, as follows. Suppose $\left(\Phi_{A}, \Phi_{X}, \Phi_{B}\right)$ : ${ }_{A} X_{B} \rightarrow M\left({ }_{C} Y_{D}\right)$, let $K$ be the linking algebra of $Y$, and define $\Phi_{L}: L \rightarrow M(K)$ by

$$
\Phi_{L}\left(\begin{array}{ll}
a & x \\
\tilde{y} & b
\end{array}\right)=\left(\begin{array}{ll}
\Phi_{A}(a) & \Phi_{X}(x) \\
\Phi_{X}(y) & \Phi_{B}(b)
\end{array}\right) .
$$

It is easy to check that $\Phi_{L}$ is then a nondegenerate homomorphism. Conversely, given such a homomorphism $\Phi_{L}$ satisfying $\bar{\Phi}_{L}\left(p_{A}\right)=p_{C}, \bar{\Phi}\left(q_{B}\right)=q_{D}$, the triple

$$
\left(p_{C} \Phi_{L}\left(\begin{array}{ll}
\cdot & 0 \\
0 & 0
\end{array}\right) p_{C}, p_{C} \Phi_{L}\left(\begin{array}{cc}
0 & \cdot \\
0 & 0
\end{array}\right) q_{D}, q_{D} \Phi_{L}\left(\begin{array}{cc}
0 & 0 \\
0 & .
\end{array}\right) q_{D}\right)
$$


is a nondegenerate imprimitivity-bimodule homomorphism. Thus we could alternatively prove Proposition 1.8 by extending $\Phi_{L}$ to a strictly continuous homomorphism $\bar{\Phi}_{L}: M(L) \rightarrow M(K)$, and applying Proposition A.1 to recover $\bar{\Phi}_{X}$ as the compression of $\bar{\Phi}_{L}$ to the top right-hand corner $M(X)$ in $M(L)$.

(3) There is a similar one-to-one correspondence between the representations $\pi=\left(\pi_{A}, \pi_{X}, \pi_{B}\right)$ of ${ }_{A} X_{B}$ on $(\mathscr{H}, \mathscr{K})$ and the representations $\pi_{L}$ of $L$ on $\mathscr{H} \oplus \mathscr{K}$, given by

$$
\pi_{L}\left(\begin{array}{ll}
a & x \\
\tilde{y} & b
\end{array}\right)=\left(\begin{array}{cc}
\pi_{A}(a) & \pi_{X}(x) \\
\pi_{X}(y)^{*} & \pi_{B}(b)
\end{array}\right) .
$$

Thus one could obtain Corollary 2.3 by applying the usual Gelfand-Naimark Theorem to $L$.

(4) Two systems $\left(A, \delta_{A}\right),\left(B, \delta_{B}\right)$ are Morita equivalent if and only if there is an $A-B$ imprimitivity bimodule $X$ and a coaction $\delta_{L}$ of $G$ on the linking algebra $L$ which compresses to the given coactions $\delta_{A}, \delta_{B}$ on the corners. (To verify this statement, one has to unravel a few hidden identifications, which are legitimate by Proposition A.1.) One can then identify $A \times_{\delta_{A}} G, B \times_{\delta_{B}} G$ and $X \times_{\delta_{X}} G$ with the corresponding corners in the crossed product $L \times{ }_{\delta_{L}} G$ : to do this, represent ${ }_{A} X_{B}$ on $(\mathscr{H}, \mathscr{K}), L \otimes C_{r}^{*}(G)$ on $(\mathscr{H} \oplus \mathscr{K}) \otimes L^{2}(G)$, and use the characterisation of the crossed product as $\overline{\mathrm{sp}}\left\{\delta_{L}(l)\left(1 \otimes M_{f}\right)\right\}$. This approach would be closer in spirit to that of Baaj-Skandalis; we have preferred to work with $X$ rather than $L$, partly because it is $X$ rather than $L$ which arises in applications, and partly because it minimises the number of identifications one has to make.

\section{REFERENCES}

1. S. Baaj and G. Skandalis, $C^{*}$-algèbres de Hopf et théorie de Kasparov équivariante, K-Theory 2 (1989), 683-721.

2. L. G. Brown, P. Green and M. A. Rieffel, Stable isomorphism and strong Morita equivalence of $C^{*}$-algebras, Pacific J. Math. 71 (1977), 349-363.

3. H. H. Bui, Morita equivalence of twisted crossed products by coactions, J. Funct. Anal. 123 (1994), 59-98.

4. R. C. Busby, Double centralizers and extensions of $C^{*}$-algebras, Trans. Amer. Math. Soc. 132 (1968), 79-99.

5. P. Green, The local structure of twisted covariance algebras, Acta Math. 140 (1978), 191-250.

6. K. K. Jensen and K. Thomsen, Elements of KK-theory, Birkhäuser, Boston - Basel - Berlin, 1991.

7. M. B. Landstad, J. Phillips, I. Raeburn and C. E. Sutherland, Representations of crossed products by coactions and principal bundles, Trans. Amer. Math. Soc. 299 (1987), 747-784.

8. K. Mansfield, Induced representations of crossed products by coactions, J. Funct. Anal. 97 (1991), $112-161$.

9. G. K. Pedersen, $C^{*}$-algebras and their automorphism groups, Academic Press, London - New York - San Francisco, 1979. 
10. J. Phillips and I. Raeburn, Twisted crossed products by coactions, J. Austral. Math. Soc. Ser. A, 56 (1994), 320-344.

11. I. Raeburn, On crossed products by coactions and their representation theory, Proc. London Math. Soc. (3) 64 (1992), 625-652.

12. M. A. Rieffel, Induced representations of $C^{*}$-algebras, Adv. in Math. 13 (1974), 176-257.

13. M. A. Rieffel, Unitary representations of group extensions; an algebraic approach to the theory of Mackey and Blattner, Studies in Analysis, Adv. Math. Supplementary Studies, Vol. 4, pp. 43-82, Academic Press, New York, 1979.

14. M. A. Rieffel, Applications of strong Morita equivalence to certain transformation group $C^{*}$-algebras, Operator algebras and applications, Proc. Sympos. Pure Math. 38 Part 1 (1982), 299-310.

FACHBEREICH MATHEMATIK-INFORMATIK UNIVERSITÄT-GESAMTHOCHSCHULE PADERBORN D-33095 PADERBORN

GERMANY
DEPARTMENT OF MATHEMATICS UNIVERSITY OF NEWCASTLE NEWCASTLE. NSW 2308 AUSTRALIA 